\title{
Embryonic Cannabidiol Exposure Does Not Affect Adult Zebrafish Swimming Performance
}

\author{
Hannah Veinot ${ }^{1}$, Erik Folkerts ${ }^{1}$, MD Ruhul ${ }^{1}$, Declam Ali ${ }^{1}$, Greg G. Goss ${ }^{1}$ \\ ${ }^{1}$ Department of Biological Sciences, University of Alberta
}

\begin{abstract}
Cannabis is used for a variety of reasons such as relieving pain, relieving stress, and reducing nausea during chemotherapy. While cannabis originates from central and south Asia, the drug has become extremely popular in North America. In July of 2001, medicinal use of cannabis was legalized in Canada, and on October 17 2018, recreational use of cannabis was legalized nationally. Many scientific studies have shown the negative effects of cannabis in consumers and of second hand smoke exposure, including lung cancer, respiratory issues, and reduced decision making and cognitive function. Because of the rapid increase in cannabis, high concentrations have filtered into the water treatment facilities and spread into lakes and ponds through pipelines that could potentially cause harm to the fish. While there are studies that have concluded that there are alterations to the fish's neuronal patterns and cardiac systems in zebrafish, there were no reports of how the medical ingredient of cannabis (cannabidiol or CBD) may affect the ability of a fish to swim. Proper swim behaviour is an essential survival characteristic to fish and other marine animals, but when a novel potentially toxic compound is introduced into their environment, impacts to vital biological functions in the organism may occur. This study aimed to investigate the potential effects of cannabidiol on zebrafish by evaluating their critical swimming speed (Ucrit value). Using a swim tunnel, we were able to control the environment and easily identify at what point the fish would be fatigued. Comparisons were made between three different fish tanks: one tank exposed to CBD, and the other two tanks contained a fresh water control and a solvent control. Using both our "p" and " $F$ " stat values, we can conclude that there were no significant differences observed between the three fish tanks. In the future, we hope to analyse the neurology of the fish exposed and complete a fish respirometry measuring the oxygen consumption of CBD exposed fish.
\end{abstract}

Key words:

embryonic, cannabidiol, exposure, zebrafish, swimming, performance, anova, aquarium, critical swimming speed, CBD, incubator

Cite as: Veinot H., Folkerts E., Ruhul MD., Ali D., Goss GG. 2019. Embryonic cannabidiol exposure does not affect adult zebrafish swimming performance. Alberta Academic Review, Vol 2 (2) 75-76, WISEST Special Issue (non peer-reviewed), DOI 10.29173/aar73. 
Veinot et al., 2019

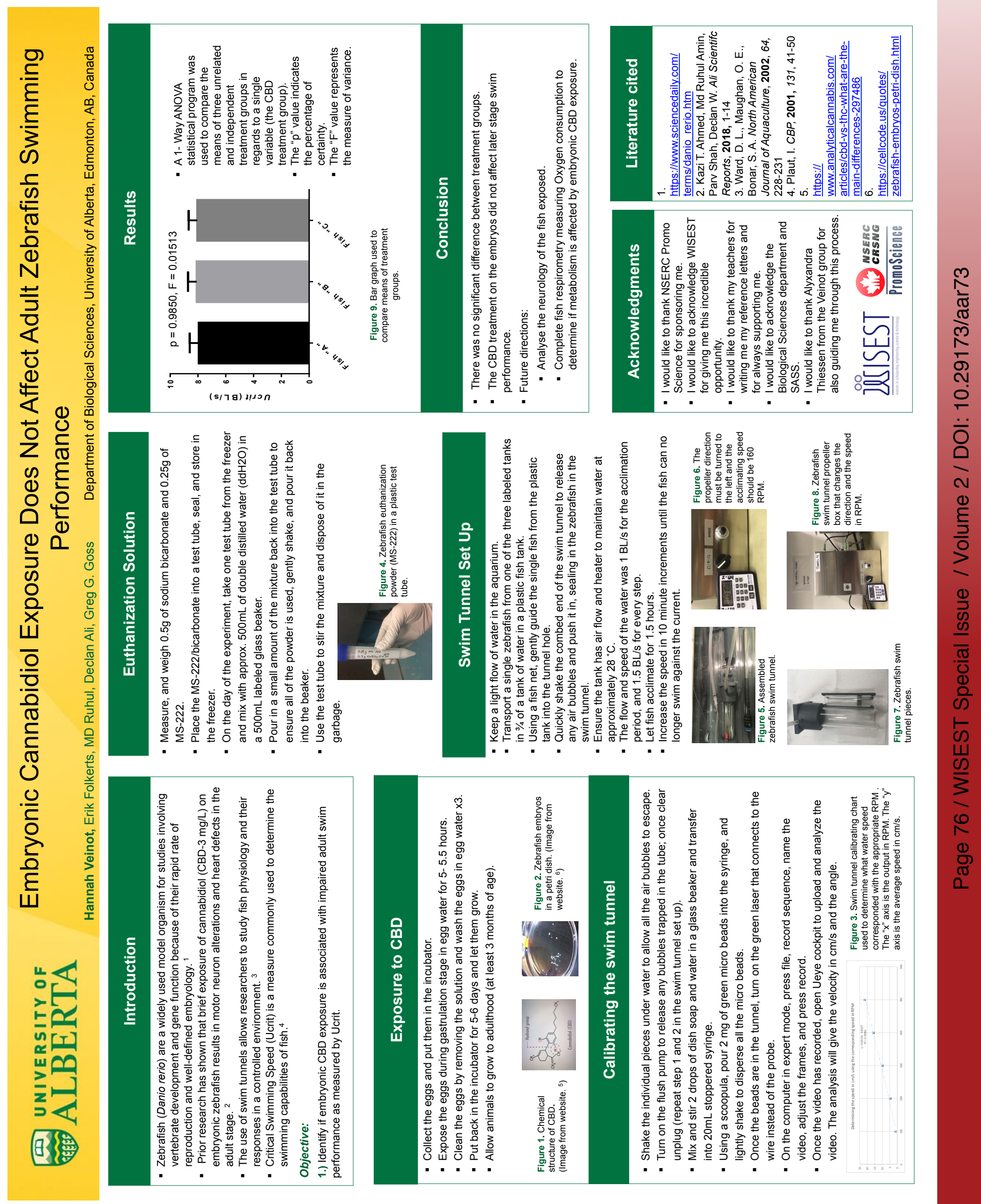

